The author is indebted to C. F. J. Outred for, among other things, the notion of rotation. The referee has pointed out that in the table for $P_{n}(=G(n) / n$ in the present notation) of [1, p. 397] the last entry should read 12198 instead of 12196 . There are further references in [1].

Department of Computer Science

University of Manchester

Manchester 13, England

1. J. Touchard, "Contributions à l'étude du problème des timbres poste," Canad. J. Math., v. 2, 1950, pp. 385-398. MR 12, 312 .

\title{
The Maxima of $P_{r}\left(n_{1}, n_{2}\right)$
}

\section{By M. S. Cheema* and H. Gupta}

1. In this note, we study the maxima of $P_{r}\left(n_{1}, n_{2}\right)$, the number of partitions of the vector $\left(n_{1}, n_{2}\right)$ into exactly $r$ parts (vectors) with positive integral components.

The generating function $\phi_{r}\left(x_{1}, x_{2}\right)$ for $P_{r}\left(n_{1}, n_{2}\right)$ is given by

$$
\begin{aligned}
\prod_{k_{1}, k_{2}=1}^{\infty}\left(1-z x_{1}{ }^{k_{1}} x_{2}{ }^{k_{2}}\right)^{-1} & =1+\sum_{r=1}^{\infty} z^{r} \phi_{r}\left(x_{1}, x_{2}\right) \\
\phi_{r}\left(x_{1}, x_{2}\right) & =1+\sum_{n_{1}, n_{2}=1}^{\infty} P_{r}\left(n_{1}, n_{2}\right) x_{1}{ }^{{ }_{1}} x_{2}{ }^{n_{2}} .
\end{aligned}
$$

2. If $q_{r}\left(n_{1}, n_{2}\right)$ denotes the number of partitions of $\left(n_{1}, n_{2}\right)$ into at most $r$ parts (vectors) with nonnegative integral components, then it follows that $q_{r}\left(n_{1}, n_{2}\right)=$ $P_{r}\left(n_{1}+r, n_{2}+r\right)$. It is clear that $q_{r}\left(n_{1}, n_{2}\right)$ is an increasing function of $r$ for $1 \leqq r$ $<n_{1}+n_{2}$, and becomes constant for $r \geqq n_{1}+n_{2}$, on the other hand $P_{1}\left(n_{1}, n_{2}\right)=1$ and $P_{r}\left(n_{1}, n_{2}\right)=0$ for $r>\min \left(n_{1}, n_{2}\right)$. From the table of values of $P_{r}\left(n_{1}, n_{2}\right)$ computed by Cheema, we notice that for $n_{1} \geqq n_{2}>0$, there is a unique $s$ such that

$$
P_{1}\left(n_{1}, n_{2}\right)<P_{2}\left(n_{1}, n_{2}\right)<\cdots<P_{s}\left(n_{1}, n_{2}\right) \geqq P_{s+1}\left(n_{1}, n_{2}\right) \geqq \cdots \geqq P_{n_{2}}\left(n_{1}, n_{2}\right) \text {. }
$$

We use $s$ in this sense in all that follows. The values of $s$ were computed for all $n_{1}$, $n_{2} \leqq 50$. We might remark that a similar conjecture holds for the number of partitions of $n$ into exactly $r$ summands. An explicit formula for $P_{r}\left(n_{1}, n_{2}\right)$ for general $r$ is not known, $P_{r}\left(n_{1}, n_{2}\right)$ do satisfy a recurrence relation and behave very much like a polynomial in $n_{1}, n_{2}$, i.e., $P_{r}\left(n_{1}, n_{2}\right)$ is a semipolynomial of degree $r-1$ in $n_{1}$ and $n_{2}$ relative to modulus $r$ ! as shown by Wright [2]. Thus

$$
\left.P_{r}\left(n_{1}, n_{2}\right)=\sum_{t_{1}=1}^{r} \sum_{t_{2}=1}^{r} \beta_{(} t_{1}, t_{2}, n_{1}, n_{2}\right) n_{1}{ }^{t_{1}-1} n_{2}{ }^{t_{2}-1},
$$

where $\beta\left(t_{1}, t_{2}, n_{1}, n_{2}\right)$ depends on $r, t_{1}, t_{2}$ and on the residues of $n_{1}, n_{2}$ to moduli 1,2 , $3, \cdots,\left[r / t_{i}\right]$, but not otherwise on $n_{1}, n_{2}$. A rough estimate for $s$ is obtained by studying the maxima of a function which behaves very much like $P_{r}\left(n_{1}, n_{2}\right)$.

Received December 7, 1966. Revised June 29, 1967.

* Supported in part by NSF under GP 7470. 
3. For $n_{1}, n_{2}$ large compared to $r, P_{r}\left(n_{1}, n_{2}\right)$ behaves very much like the function

$$
\frac{1}{r !}\left(\begin{array}{c}
n_{1}-1 \\
r-1
\end{array}\right)\left(\begin{array}{c}
n_{2}-1 \\
r-1
\end{array}\right) \text {. }
$$

Using this estimate and using $P_{r}\left(n_{1}, n_{2}\right) \geqq P_{r+1}\left(n_{1}, n_{2}\right)$, we obtain $s=\min \left(r, n_{1}, n_{2}\right)$, where $r$ is the least positive integer satisfying

$$
\left(n_{1}-r\right)\left(n_{2}-r\right) \leqq r^{2}(r+1) \text {. }
$$

Roughly such an $r$ is given by $\left(n_{1} n_{2}\right)^{1 / 3}$. If $n_{1}=n_{2}=n$, then as in [1]

$$
P_{r}(n, n) \simeq \frac{1}{r !}\left(\begin{array}{c}
n-1 \\
r-1
\end{array}\right)^{2} \exp \left(\frac{r^{3} \operatorname{og} r}{n^{2}}\right) .
$$

Hence $P_{s}(n, n) \geqq P_{s+1}(n, n)$ implies that

$$
(n-s)^{2} \leqq(s+1) s^{2-\left(3 s^{2}+3 s+1\right) / n 2} .
$$

As a rough estimate we have $s \simeq n^{2 / 3}$. The inequality (3.3) gives a good estimate for $s$ for a particular $n$. Thus for $n=50$, the value of $s$ by (3.3) is 14 , while the actual value is 13 . For $n=52, s=14$ both by the inequality and the tables.

The University of Arizona

Tucson, Arizona

1. H. Gupta, "An inequality for $P\left(N_{j}, k\right)$," Res. Bull. Panjab Univ., v. 13, 1962, pp. 173-178. MR $28 \# 58$.

2. E. M. Wright, "Partitions of multipartite numbers into a fixed number of parts," Proc. London Math. Soc. (3), v. 11, 1961, pp. 499-510. MR 24 \#A2573. 